\title{
Results from MuSiCoS Campaigns on Be stars
}

\author{
H.F. Henrichs \\ Astronomical Institute, University of Amsterdam, Kruislaan 403, \\ 1098SJ Amsterdam, Netherlands \\ C. Neiner, A.M. Hubert, M. Floquet \\ Observatoire de Meudon, France
}

and MuSiCoS Team

\begin{abstract}
The contribution of the MuSiCoS project (a multisite network of $2 \mathrm{~m}$ class telescopes) to Be-star research is summarized. Some preliminary results of the 1998 campaign on $\omega$ Ori are shown.
\end{abstract}

\section{Introduction}

MuSiCoS is an international project for a multisite network of high-resolution spectrometers around the world for Multi Site Continuous Spectroscopy. A major scientific goal of $\mathrm{MuSiCoS}$ is to measure stellar non-radial pulsations on $\mathrm{OB}, \mathrm{Be}, \delta \mathrm{Scuti}$, fast rotating and solar-type stars. The project was initiated in 1988 by Catala and colleagues. A transportable high-resolution $(R=30000)$ fiberfed spectrograph, dedicated to this project, was completed in 1991 (Baudrand and Böhm 1992, Catala et al. 1993). This first MuSiCoS spectrograph has its home base at the Telescope Bernard Lyot at Pic du Midi (France), but has been used at several sites at the Northern and Southern hemisphere. The spectrograph has been duplicated a few times, for instance the ESA MuSiCoS spectrograph, which is based at the INT at La Palma.

A dedicated polarimeter attached to the first MuSiCoS spectrograph is available (Donati et al. 1999), and has produced excellent results in detecting weak magnetic fields on the surfaces of a wide variety of stars, including some B stars. (See for example Henrichs, these proceedings).

\section{Be stars with MuSiCoS: $\boldsymbol{\omega}$ Ori in 1998}

The paper by Hao (these proceedings) gives an complete summary of Be star observations (and other objects) in the MuSiCoS collaboration. As part of the 1998 campaign 3 Be stars were observed: $\omega$ Ori, $\epsilon$ Per and $\psi^{2}$ Ori. As the reduction and analysis of such a diverse array of telescopes and spectrographs is non-trivial, it will take some time to complete the individual projects. We list in Table 1 (prepared by the PI of this project, A.M. Hubert) the log of observations with the used instrumentation and observers of the campaign on the B3 IIIe star $\omega$ Ori. 


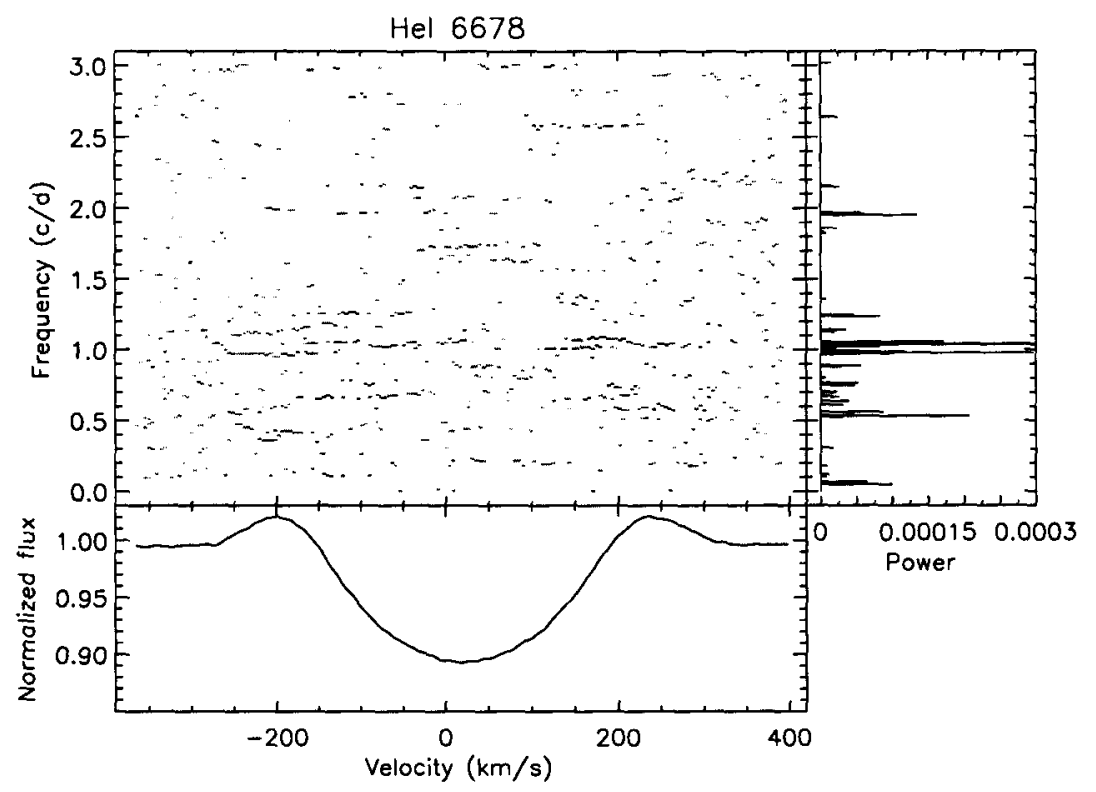

Figure 1. A preliminary frequency analysis of the He I 6678 line of normalized spectra of $\omega$ Ori collected as part of the $1998 \mathrm{MuSiCoS}$ campaign during November 23-29 (from Neiner et al., in preparation). The level of grey is proportional to the power. A number of significant frequencies across the line profile can be identified, but confirmation has to come from a comparison with similar diagrams for other spectral lines. The summed power is given in the righthand panel, whereas the average profile is given in the lower panel.

\section{Line profile analysis}

Several lines were used to search for $l p v$ : He I 4471, 5876, 6678, Si III triplet $4553,4568,4575$, and $\mathrm{H} \alpha$. We applied different methods, especially the Restricted Local Cleanest (RLC) (see de Souza Jr, 1999). The S/N ratio measured in the continuum next to the line is used to assign a weight to each spectrum. In each wavelength bin, the RLC method searches for 30 frequencies in a predefined range from which it computes all possible models with 4 frequencies. Comparing the power of each of these models, it selects the 7 optimal frequencies, while suppressing the aliases. A local cleanest method (see Foster, 1995) is then applied to these 7 values, i.e. the RLC method looks for 7 points around each of these frequencies to finetune the final frequencies. By looking carefully at the window spectrum, we can remove the window aliases that may remain. Periods longer than the total duration of the observing run of 22 days $(\nu \leq 0.047 \mathrm{c} / \mathrm{d})$ cannot be detected. The frequency resolution is around $0.01 \mathrm{c} / \mathrm{d}$.

As a first result we show in Fig. 1 the variability of $\omega$ Ori in the He I 5876 line, collected at OHP during November 23-29, 1998 (from Neiner et al. in preparation). These results are based on the analysis of 171 spectra (weighted ac- 
Table 1. Observations of $\omega$ Ori, MuSiCoS 1998, 20 Nov, - 13 Dec.

\begin{tabular}{llllll}
\hline Sites & OHP & OHP & INT & ESO & KP \\
\hline Aperture & $1.52 \mathrm{~m}$ & $1.93 \mathrm{~m}$ & $2.5 \mathrm{~m}$ & $0.9 \mathrm{~m}$ & $0.9 \mathrm{~m}$ \\
Spectrogr. & Aurélie & Elodie & MuSiCoS & HEROS & Echelle \\
Resol. & 22000 & 43000 & 35000 & 20000 & 65000 \\
$\lambda \lambda(\mathrm{nm})$ & $652-672$ & $390-700$ & $400-670$ & $350-870$ & $530-700$ \\
Runs & $20 / 11-2 / 12$ & $23-30 / 11$ & $2-10 / 12$ & $25 / 11-12 / 12$ & $23 / 11-3 / 12$ \\
Spectra & 33 & 29 & 36 & 47 & 33 \\
Obs. & Orlando & Neiner & Abbott & Schaefer & Neff \\
& Cami & & Telting & Tubbesing & O'Neal \\
& & & & & \\
\hline Sites & XL & LNA & Mt Stromlo & SAO & SAAO \\
\hline Aperture & $2.16 \mathrm{~m}$ & $1.6 \mathrm{~m}$ & $1.9 \mathrm{~m}$ & $1 \mathrm{~m}$ & $1.9 \mathrm{~m}$ \\
Spectrogr. & Echelle & Coudé & Echelle & Echelle & GIRAFFE \\
Resol.n & 35000 & 60000 & 35000 & 45000 & 20000 \\
$\lambda \lambda(\mathrm{nm})$ & $445-672$ & $663-672$ & $480-680$ & $350-1000$ & $430-691$ \\
Runs & $28 / 11-7 / 12$ & $5-10 / 12$ & $29 / 11-3 / 12$ & $29 / 11-4 / 12$ & $1-4 / 12$ \\
Spectra & 22 & 27 & 13 & 1 & 2 \\
Obs. & Cao & Souza & Baldry & Galatzudinov & Buckley \\
& Orlando & & O'Toole & Musaev & \\
\hline
\end{tabular}

cording to the reached S/N per unified velocity bin) from Mt Stromlo, OHP152, Xinglong, HEROS, LNA and INT.

\section{Prospect}

At the present stage it is too early to draw conclusions from the preliminary analysis. Once all 242 spectra of the 10 different telescopes will be reduced to a set with homogeneous quality, the data will be subject to a Fourier analysis with several methods to search for non-radial pulsations. Special attention will be given to purely photospheric lines (blue He I, Si III, Si II, Al II, C II, O II, $\mathrm{Mg}$ II), the red He lines affected by an near-photospheric contribution, and the main Balmer lines, which were all well covered.

Acknowledgments. We thank the many participants (see Table 1) of the MuSiCoS campaign for an always pleasant and fruitful collaboration.

\section{References}

Baudrand, J., Böhm, T. 1992, A\&A 259, 711

Catala, C. Foing, B.H., Baudrand, J. et al. 1993, A\&A 275, 245

de Souza Jr, D. 1999, preprint

Donati, J.-F., Catala, C., Wade, G. et al. 1999, A\&AS 134, 149

Foster, G. 1995, AJ 109, 1889 